\title{
Analysis of Factors Leading to Chronic Atrophic Gastritis and Clinical Treatment
}

\author{
Ling Zhang \\ Luohe Medical College, Luohe, Henan, 462000
}

Keywords: Chronic atrophic gastritis; risk factors; clinical treatment

\begin{abstract}
Objective: To explore the risk factors and clinical treatment effects of patients with chronic atrophic gastritis. Methods: 98 patients with chronic atrophic gastritis were selected as the observation group, and 100 patients with chronic non-atrophic gastritis were selected as the control group. The risk factors of the two groups were statistically analyzed. In addition, 54 patients who were positive for Helicobacter pylori (Hp) in the observation group were randomly divided into two groups, group A and group B, given group A triple therapy + teprenone, group B received triple therapy + teprenone + Folic acid treatment, and compare the clinical efficacy of the two groups. Results: The risk factors of patients with chronic atrophic gastritis mainly include age, family history of gastric cancer, and reflux of bile. In addition, the total effective rate of treatment in group $\mathrm{B}$ was significantly higher than that in group A $(\mathrm{P}<0.05)$. Conclusions: Senior age, family history of gastric cancer, and reflux of bile are risk factors for chronic atrophic gastritis. However, patients with Hp-positive therapy can achieve the desired therapeutic effect by folic acid-based combination therapy.
\end{abstract}

\section{Introduction}

As a common digestive system disease in clinic, the main features of chronic atrophic gastritis are thinning of gastric mucosa, gastric mucosa gland and epithelial atrophy. The clinical symptoms of these patients are mainly nausea, loss of appetite and abdominal pain. They have a high recurrence rate and cancer rate. If scientific and effective treatment is not adopted, the probability of developing gastric cancer is as high as $20 \%$ or more. Health and quality of life have had a very serious impact. Based on this, this paper explores the risk factors and clinical treatment effects of patients with chronic atrophic gastritis, in order to promote the improvement of clinical treatment level. The specific operation is as follows.

\section{Materials and methods}

General Information: 98 patients with chronic atrophic gastritis admitted to the First Affiliated Hospital of Luohe Medical College from February 2015 to March 2017 were observed in the observation group, including 52 males and 46 females; the average age was $634 \pm 6.7$ ) years old; Another 100 patients with chronic non-atrophic gastritis were selected as the control group, 53 males and 47 females; the average age was (63. $8 \pm 7.1$ ) years old. All patients were diagnosed by clinical pathology, and patients with early diseases such as severe heart, liver and kidney, gastric ulcer and stomach cancer were excluded. In addition, 54 patients who were positive for Hp in the observation group were randomly divided into two groups, A and B, with 27 patients in each group. There was no significant difference in the data between the control group and the observation group, group A and group B ( $\mathrm{P}>0.05)$, which was comparable.

Methods: 1 Risk factor analysis: Retrospective analysis was performed on the age, sex, smoking history, family history of gastric cancer, and Helicobacter pylori (Hp) infection in the observation group and the control group. 2 Therapy: A group of triple therapy + teprenone is given, the specific operation is: oral esomeprazole, $0.02 \mathrm{~g} /$ time, 2 times / d; oral clarithromycin, $0.5 \mathrm{~g} / \mathrm{d}$, 2 times / $\mathrm{d}$; oral amoxicillin, $0.1 \mathrm{~g} /$ time, 2 times / d. Teprenone was orally administered $150 \mathrm{mg}$ once daily for 3 times / d. Group B received triple therapy + teprenone + folic acid treatment, which was based 
on group A treatment plus $10 \mathrm{mg}$ folic acid, 3 times / d. 10 days was a course of treatment and both groups received a 12-week treatment.

Efficacy criteria: Healing: The clinical symptoms of the patients disappeared, and the gastric mucosa returned to normal after gastroscopy. Significant effect: The clinical symptoms were significantly improved, the gastric mucosa was mainly red, and the intrinsic gland atrophy disappeared. Effective: Clinical symptoms Improvement, gastroscopy revealed vascular streaks and gland atrophy disappeared; Ineffective: Clinical symptoms and glandular atrophy did not change, and even worsened.

Statistical processing: The SPSS13.0 software was used to statistically analyze and analyze all the data, and the composition ratio between the groups was tested. When there was a significant difference between the groups, that is, $\mathrm{P}<0.05$, the study was statistical. significance.

\section{Results}

Analysis of risk factors: According to statistical analysis, the risk factors of patients with chronic atrophic gastritis mainly include age, family history of gastric cancer, reflux of bile, etc. $(\mathrm{P}<0.05)$. See Table 1.

Table 1. Comparisonss of risk factors between patients with chronic atrophic and non-atrophic gastritis

\begin{tabular}{|c|c|c|c|c|}
\hline \multicolumn{2}{|l|}{ Factors } & Observe(n=98) & $\begin{array}{ll}\text { Control } & \text { group } \\
(n=100) & \end{array}$ & $\mathrm{P}$ \\
\hline \multirow[t]{2}{*}{ Sex } & Male & 52 & 53 & \multirow[t]{2}{*}{$>0.05$} \\
\hline & Female & 46 & 47 & \\
\hline \multirow{2}{*}{$\begin{array}{ll}\text { Family } & \text { history } \\
\text { of } & \text { gastric } \\
\text { cancer } & \end{array}$} & Yes & 37 & 18 & \multirow[t]{2}{*}{$<0.05$} \\
\hline & No & 61 & 82 & \\
\hline \multirow[t]{2}{*}{ Smoking or not } & Yes & 45 & 47 & \multirow[t]{2}{*}{$>0.05$} \\
\hline & No & 53 & 53 & \\
\hline \multirow[t]{2}{*}{ Drinking or not } & Yes & 33 & 36 & \multirow[t]{2}{*}{$<0.05$} \\
\hline & No & 65 & 64 & \\
\hline \multirow{2}{*}{$\begin{array}{l}\text { Bile with or } \\
\text { without reflux }\end{array}$} & Yes & 21 & 8 & \multirow[t]{2}{*}{$>0.05$} \\
\hline & No & 77 & 92 & \\
\hline \multirow[t]{2}{*}{ Hp infection } & Yes & 72 & 43 & \multirow[t]{2}{*}{$<0.05$} \\
\hline & No & 26 & 57 & \\
\hline
\end{tabular}

Table 2 Comparison of therapeutic effects between two groups of patients with chronic atrophic gastritis

\begin{tabular}{|l|l|l|l|l|l|l|}
\hline Gr & Case & get well & $\begin{array}{l}\text { Very } \\
\text { Effective }\end{array}$ & Effective & $\begin{array}{l}\text { None } \\
\text { Effective }\end{array}$ & Total Rate \\
\hline Gr. A & 27 & 7 & 9 & 5 & 6 & $77.8 \%$ \\
\hline Gr. B & 27 & 11 & 8 & 6 & 2 & $92.6 \%$ \\
\hline
\end{tabular}

Chronic atrophic risk Hp positive treatment effect: After a period of treatment, the total effective rate of treatment in group B was $92.6 \%$, significantly higher than $77.8 \%$ in group $\mathrm{A}$, the data difference was statistically significant. Meaning $(\mathrm{P}<0.05)$. See Table 2.

\section{Discussion}

Chronic atrophic gastritis is a chronic inflammatory reaction. For severe patients, it will have a series of complications in the late stage of the disease, such as intestinal metaplasia, dysplasia, etc., and some patients will also evolve into gastric cancer, which is known as Effective prevention of the stomach 
In the occurrence of cancer, clinical medical staff should conduct an in-depth analysis of the risk factors and clinical features of chronic atrophic gastritis. The results of this study indicate that the risk factors for patients with chronic atrophic gastritis include age, family history of gastric cancer, bile reflux, and Hp infection. The following are some of the risk factors: 1 Old age: As a typical risk factor for many diseases, it is old age. This is because for older patients, there are more basic diseases, and their own immunity is not high. Some of the worsening of the primary disease provide favorable conditions; in addition, the elderly patients have a longer course of disease, leading to disease progression. 2 family history of gastric cancer: Many stomach diseases have an indirect or direct relationship with the genetic history of gastric cancer. For patients with a family history of gastric cancer, the genetics of this disease exists chronically compared with the general population. The probability of atrophic gastritis is greater. 3 Bile reflux: When the patient's pyloric sphincter relaxes, the bile in the duodenum will flow back to the stomach, causing a stimulating effect on the stomach and causing gastritis. In addition, bile also contains bile salts, which can damage the gastric mucosa, leading to the occurrence of secondary atrophic gastritis. 4Hp infection: Helicobacter pylori (Hp) is easy to cause imbalance between the body's defense factor and attack factor. For patients with long-term Hp infection, the probability of intestinal mucosal intestinal metaplasia and atrophy is greater.

In this study, 54 patients with chronic atrophic gastritis who were positive for Hp were taken as an example to explore their treatment methods. Considering that antibiotic resistance has been increasing in recent years, triple therapy alone cannot achieve satisfactory clinical results.

Folic acid is added to the patient. Folic acid mainly includes the cost of acridine and glutamic acid, which can stimulate the proliferation of the digestive tract epithelium, and can promote the synthesis and methylation of DNA and protein, thereby inhibiting the expression of cellular genes. The results of the experiment showed that the total effective rate of treatment in group B patients with folic acid-based treatment was significantly higher than that in group A patients with triple therapy $(\mathrm{P}<0.05)$. This result is consistent with the research report of Wang Zhongluo and others.

In summary, advanced age, family history of gastric cancer, and reflux of bile are risk factors for chronic atrophic gastritis. However, f-acid-based combination therapy is effective in patients with positive Hp.

\section{References}

[1] Chen Meiqing, Li Mingchun. Analysis of risk factors for recurrence of chronic atrophic gastritis[J]. World Medical Information Digest, 2015, 15( 94) : 300.

[2] Li Tianhua. Clinical experience of digestive medicine in patients with chronic atrophic gastritis [J]. Health, 2014, 8 ( 14) : 125.

[3] Zhu Jinsheng, Shi Wei. Study on the effect of standardized treatment of digestive medicine in patients with chronic atrophic gastritis[J]. Journal of Contemporary Medicine, 2014, 12( 21) : 254.

[4] Zhang Yan, Lu Guangxin. Therapeutic effect of Yangweishu combined with folic acid on chronic atrophic gastritis[J]. Anhui Medical Journal, 2013, 17( 04) : 666.

[5] Liu Junjie, Zhang Hongyan, Zhang Fengmei, et al. Clinical treatment of gastroenterology in patients with chronic atrophic gastritis[J]. Chinese Medicine Guide, 2015, 13(13) : 165.

[6] Peng Qingjuan, Cai Keyin. Progress in the treatment of chronic atrophic gastritis with traditional Chinese medicine and western medicine[J]. Journal of Zhejiang University of Traditional Chinese Medicine, 2013, 37( 3) : 359.

[7] Zhai Yaojun. Clinical analysis of the treatment of chronic atrophic gastritis with integrated traditional Chinese and Western medicine[J]. Journal of Contemporary Medicine, 2011, 19(3): 145.

[8] Wang Zhongluo, Zhou Gang, Shen Huilin. Risk factors and clinical treatment of patients with chronic atrophic gastritis[J]. Chinese Journal of Nosocomiology, 2015, 25(8): 1817. 